\title{
PAPER
}

\section{Dermal microdialysis provides evidence for hypersensitivity to noradrenaline in patients with familial dysautonomia}

\author{
A Bickel, F B Axelrod, M Schmelz, H Marthol, M J Hilz
}

J Neurol Neurosurg Psychiatry 2002;73:299-302

See end of article for authors' affiliations

..................

Correspondence to: Dr M J Hilz;

max.hilz@neuro.imed. uni-erlangen.de

Received

7 November 2001

In revised form 7 May

2002

Accepted 20 May 2002

\begin{abstract}
Objectives: To use the technique of dermal microdialysis to examine sensitivity of skin vessels to noradrenaline (NA) in patients with familial dysautonomia (FD) and in healthy controls.

Methods: In 14 patients with FD and 12 healthy controls, plasma extravasation, local laser Doppler blood flow, and skin blanching were observed before, during, and after application of $10^{-6} \mathrm{MNA}$ through a microdialysis membrane, located intradermally in the skin of the lower leg.

Results: Maximum local vasoconstriction measured by laser Doppler blood flow did not differ between patients with FD and controls. In contrast, patients with FD had an earlier onset of vasoconstriction $(p=0.02)$. Moreover, reaction to NA was more prominent and prolonged in FD, shown by a larger zone of skin blanching around the microdialysis membrane $(p<0.001)$ and delayed reduction of the protein content in the dialysate after termination of NA application ( $p=0.03$ ).

Conclusion: These data support the hypothesis that peripheral blood vessels of patients with FD show a denervation hypersensitivity to catecholamines. This may be one mechanism contributing to the major hypertension that frequently occurs during "dysautonomic crises" in FD.
\end{abstract}

A utonomic dysfunction is the principal symptom of patients with familial dysautonomia (FD), a genetic disorder characterised by decreased populations of sensory, sympathetic, and parasympathetic neurones. ${ }^{1}$ Prominent losses of sympathetic neurones in ganglia ${ }^{2}$ and in peripheral blood vessels ${ }^{4}$ have been described in neuropathological studies and have been suggested to contribute to clinical signs of cardiovascular instability including blood pressure lability. Patients with FD exhibit orthostatic hypotension as well as episodic hypertensive "dysautonomic" crises with peripheral vasoconstriction, acrocyanosis, excessive sweating, and piloerection following emotional stress. ${ }^{5}$ It has been assumed that dysautonomic crises are caused by hypersensitivity to catecholamines.

However, this theory is discussed controversially in the literature. Upregulation ${ }^{67}$ and dysfunction of peripheral $\alpha$ adrenoceptors ${ }^{8}$ have been suggested as possible explanations for the clinical symptoms. Experiments to prove this hypothesis are difficult to perform. Major hypertension in FD patients, which is induced by systemic infusion of catecholamines, ${ }^{6}$ may in part be attributed to altered central blood pressure regulation. Other techniques to deliver noradrenaline (NA) to skin blood vessels by injection or iontophoresis also may cause increased sympathetic outflow by an arousal reaction and axon reflex vasodilatation by excitation of afferent nociceptal C fibres. Furthermore, injection or iontophoretic application of NA may change local perfusion and thus interfere with NA induced vasoconstriction. In contrast, application of NA by microdialysis is non-painful and therefore avoids these biasing influences and facilitates analysis of vascular reactions.

In our study we used the technique of dermal microdialysis to deliver atraumatically a defined dose of NA intradermally and to measure vasoconstriction of local blood vessels. Only initial insertion of the needles causes moderate pain, whereas ensuing microdialysis is painless. NA application was not started earlier than 30 minutes after membrane insertion.

In case of hypersensitivity to catecholamines in patients with FD, we would expect a prolonged or more accentuated vasoconstriction to NA application. To test this hypothesis, we used microdialysis not only for NA application but also simultaneously to measure protein concentration of interstitial fluid as a parameter of vascular permeability and local capillary blood flow. Local skin reactions were analysed by measuring the area of local vasoconstriction ("blanching") around the NA membrane and registering changes of the local capillary blood flow by laser Doppler flowmetry.

\section{MATERIALS AND METHODS}

Fourteen patients with FD (six men and eight women, age range 18-45, mean (SEM) 22.8 (3.2) and 34.5 (2.9) years, respectively) were studied at the Dysautonomia Treatment and Evaluation Center at New York University Medical Center. All patients with FD fulfilled accepted clinical diagnostic criteria: Ashkenazi Jewish ancestry and absence of deep tendon reflexes, overflow tears, lingual fungiform papillae, and axon flare response following intradermal histamine injection. ${ }^{5}$ In addition, all patients had the most common genetic haplotype-that is, they were homozygous for the intron 20 mutation on the IKBKAP gene.' All patients gave their written consent to participate in the study. The protocol was approved by the Institutional Review Board of New York University Medical Center. All patients were asked to discontinue their medication, including fludrocortisone and midodrine, for 18 hours before testing. Patients who were not able to comply with this requirement were not included in the study.

Subjects were seated comfortably in a near supine position, with only the head lifted by $30^{\circ}$ or less. We also studied 12 healthy age and sex matched volunteers (six men and five women, age range 22-37, mean (SEM) 26.5 (1.9) and 29.4 (2.3) years, respectively) as controls.

Abbreviations: FD, familial dysautonomia (Riley-Day syndrome); NA, noradrenaline; $\mathrm{pU}$, perfusion units; SBF, skin blood flow; TPC, total protein content 


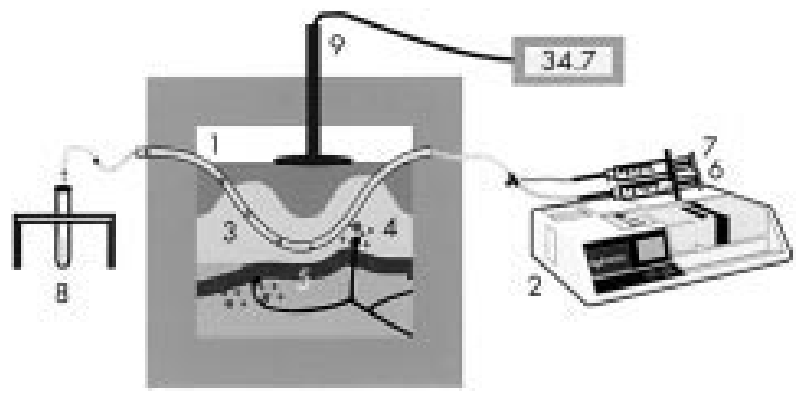

Figure 1 Setup of the microdialysis experiment. The semipermeable microdialysis membrane (1) is connected to a microdialysis pump (2) and inserted intracutaneously (3) close to free intracutaneous nerve endings (4) and subcutaneous blood vessels (5) and is perfused alternately with Ringer's solution (6) and Ringer's solution containing $10^{-6} \mathrm{M}$ noradrenaline (NA) (7). Substances and molecules as large as plasma proteins can circulate freely between interstitial tissue and the microdialysis fluid, which is collected in polyethylene cups for further analysis after skin passage (8). A laser Doppler probe (9) is fixed tightly to the skin above the microdialysis membrane and laser Doppler flux values are monitored continuously.

For microdialysis, the skin at the lower leg above the tibial anterior muscle was consistently used as the test site. Hollow plasmapheresis fibres $(0.4 \mathrm{~mm}$ diameter, cut off $3000 \mathrm{kDa}$, Asahi, Tokyo, Japan) were inserted intracutaneously at a length of $1.5 \mathrm{~cm}$ in the skin by means of a 25 gauge canula. All membranes were inserted transversely orientated about $10 \mathrm{~cm}$ proximal the ankle region. The applied technique ${ }^{10}$ ensured a strictly intracutaneous position of the membranes with an insertion depth between 0.4 and $0.9 \mathrm{~mm}$. Local anaesthesia was not necessary, as insertion of the membranes was only slightly painful. After insertion, the fibre was connected through a Tygon tube (Novodirect, Kehl, Germany) to a syringe in a microdialysis pump (No 540230, TSE GmbH, Bad Homburg, Germany) containing Ringer's solution. Perfusion was started immediately after insertion of the membrane and continued throughout the whole experimental session of 90 minutes at a flow rate of $4 \mu \mathrm{l} / \mathrm{min}$. After a baseline period of 30 minutes, the microdialysis membrane was connected to a second syringe containing $10^{-6} \mathrm{M}$ NA in Ringer's solution for another 30 minutes and then switched back to Ringer's solution without NA. After skin passage the dialysate was collected in polyethylene cups attached to the skin. Samples were taken every 15 minutes for 120 minutes. All samples were frozen immediately after the test session and stored at $-20^{\circ} \mathrm{C}$ or lower until analysed.

For direct evaluation of vascular responses a laser Doppler flowmeter (Periflux, Perimed, Stockholm, Sweden) was used. The laser probe was located exactly over the microdialysis membrane and fixed firmly to the skin. Laser Doppler flux, expressed as arbitrary perfusion units (pU), was monitored continuously throughout the experimental session. Additionally, the area of vasoconstriction during NA application (blanching of the skin) was measured 15 and 30 minutes after the beginning of NA application. Maximum diameters to both sides of the microdialysis membrane were used for further evaluation. Figure 1 summarises the experimental setup.

To determine the total protein content (TPC) of the microdialysis samples, we used the Coomassie blue method according to Bradford. ${ }^{11}$ After addition of the test reagent, protein content was analysed photometrically using an MRX reader (Dynatech, Rückersdorf, Germany).

\section{Statistical analysis}

To assess responses to exogenously applied catecholamines, we compared differences between the patient and control groups using Student's $t$ test. Values are given as mean (SEM). Significance was set at $\mathrm{p} \leqslant 0.05$.

\section{RESULTS}

Laser Doppler flow during experimental stages

Initial insertion of the microdialysis membranes induced a transient neurogenic and non-neurogenic erythema of the skin, which is caused by the insertion trauma as well as activation of afferent $C$ fibres. The initial local skin reaction was less pronounced in the patient group than in the controls. Laser Doppler monitoring of the skin blood flow (SBF) was started after membrane insertion and continued for the 30 minute baseline measurement. Although the initial values were significantly higher in controls $(38.1(8.5) \mathrm{pU})$ than in patients with FD (18.2 (4.3) pU, p=0.03), they decreased quickly in both groups. At the end of the 30 minute baseline period, local SBF measured by laser flowmetry was consistently lower in patients (13.3 (4.0) pU) than in controls (26.5 (7.0) $\mathrm{pU}$ ) without reaching significance $(\mathrm{p}=0.11)$.

Intracutaneous application of $10^{-6} \mathrm{M}$ NA was started 30 minutes after insertion of the microdialysis membrane and induced a rapid decrease of local perfusion. SBF reached a minimum in controls $(5.0(2.5) \mathrm{pU})$ and in patients (2.1 (l.1) pU) after approximately 15 minutes of NA infusion. After 30 minutes of NA application, perfusion of the microdialysis membrane was switched back to Ringer's solution without NA. During this period, SBF recovered slowly but did not reach baseline values in either group. Until the end of the experiment, values still were significantly lower in patients (3.4 (0.4) pU) than in controls $(6.8(3.5) \mathrm{pU}, \mathrm{p}=0.003)$.

To compare fluxes between patients and controls, data were normalised to baseline values immediately before starting NA microdialysis. Normalisation showed that SBF dropped to values about $75 \%$ lower than baseline values in both groups during NA application and differences in SBF values were not significant. However, the time course of SBF changes differed between patients and controls. In patients with FD, SBF values declined rapidly and immediately after the beginning of NA application, while in controls SBF values remained stable for at least five minutes and started to decline only afterwards. Additionally, the first normalised SBF value taken after the start of NA application differed significantly between both groups $(64.8 \% \vee 100.0 \%, \mathrm{p}=0.02$; fig 2$)$.

\section{Skin blanching with NA induced vasoconstriction}

An area of skin blanching developed symmetrically to both sides of the microdialysis membrane after onset of NA infusion with sharp borders to the surrounding skin. The size of the blanching area was marked on the skin in 10 minute intervals. The maximal size that was reached during the second half of the 30 minute stimulation period was transferred to a transparency sheet and used for further analysis. The diameter of the blanching zone was significantly larger in the patient group $(8.9(0.5) \mathrm{mm})$ than in the control group $(6.5(0.3) \mathrm{mm}, \mathrm{p}<0.001)$.

\section{Protein content of the dialysate}

Microdialysate was sampled at 15 minute intervals so that two samples were obtained during the 30 minute baseline, two during 30 minutes of NA stimulation, and two during the 30 minute washout after termination of the NA stimulation.

Probe volume per sample was $60 \mu \mathrm{l}$, according to the flow rate of $4 \mu \mathrm{l} / \mathrm{min}$. The TPC of each of the six dialysate probes was evaluated.

In both groups, TPC was increased in the probe sampled during the first 15 minutes after membrane insertion, which was caused by positioning induced tissue damage. In the second probe TPC decreased significantly in both groups. Previous experiments on microdialysis showed that a stable baseline concentration of plasma extravasation is reached about 30 minutes after membrane insertion. ${ }^{12}$

During NA application, TPC decreased further in the dialysate of patients and controls, but did not differ between 


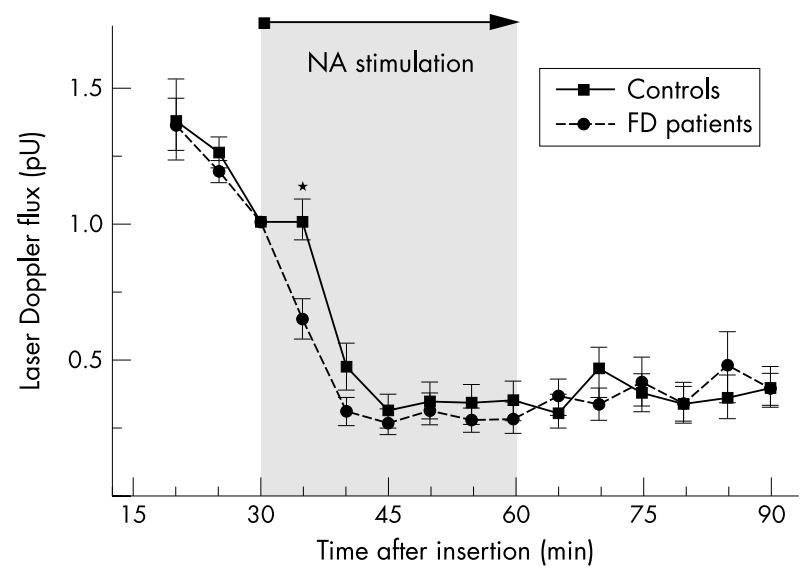

Figure 2 Laser Doppler flux (measured in arbitrary perfusion units (pU)) differs only during the wash-in of NA. Skin blood flow (SBF) rates were measured by a laser Doppler probe attached to the skin directly above the microdialysis membrane and normalised to baseline values. The baseline period (0-30 minutes) was followed by NA microdialysis (30-60 minutes) and a washout phase (60-90 minutes). During NA application SBF decreased by approximately $75 \%$ of baseline values in both groups, but patients with familial dysautonimia (FD) responded faster and therefore to lower local concentrations of NA. Minimum SBF was not different between patients with FD and controls, suggesting that the chosen dose of NA was high enough to produce maximum vasoconstriction at the application site. There were also no significant differences in SBF during the washout phase. ${ }^{*} p=0.02$.

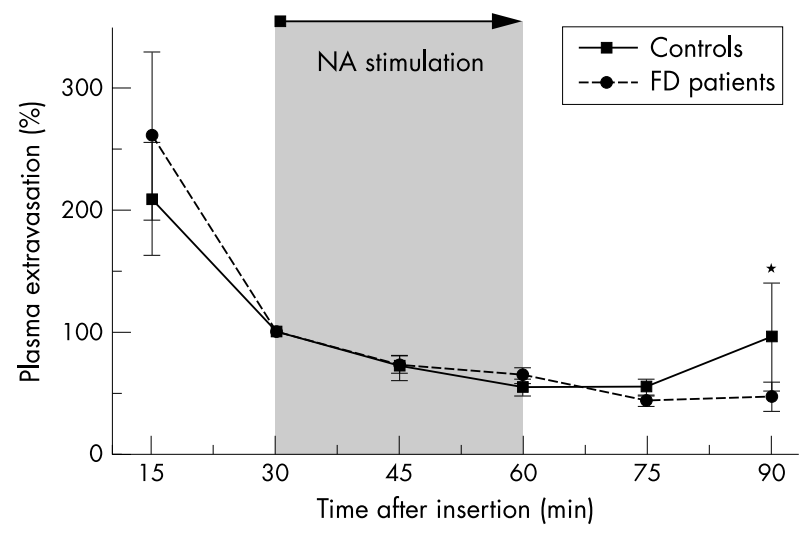

Figure 3 Effects of NA application on plasma extravasation. After insertion of the microdialysis membrane, interstitial protein decreased within 30 minutes in both controls and patients with FD. During NA application (30-60 minutes) protein content did not differ between samples of the controls and the patients. After termination of the NA application (60-90 minutes) protein concentrations in the samples returned to baseline values in the controls but remained low in the patients, suggesting that the vasoconstrictor effect of small doses of NA was prolonged in patients with FD. ${ }^{*} p=0.03$.

the groups. Within 30 minutes after NA application, TPC increased in controls up to $94 \%(0.0067 \mathrm{~g} / \mathrm{l})$ of baseline concentrations but remained at $45 \%(0.002 \mathrm{~g} / \mathrm{l})$ of baseline values in the patients ( $p=0.03$; fig 3$)$.

\section{DISCUSSION}

In our study, the minimally invasive technique of dermal microdialysis was used for the first time in patients with FD to atraumatically deliver NA intradermally and to assess local reactions of peripheral blood vessels. This method provides several advantages over conventional means of NA application such as infusion or iontophoresis. In contrast to systemic NA administration, ${ }^{6}$ microdialysis has few associated complications, as the vasoactive agent is applied only locally to a very small skin area. Furthermore, the application is painless and therefore avoids generalised sympathetic arousal or activation of local pain mediating afferent $\mathrm{C}$ fibres, which may significantly influence experimental results.

The microdialysis membrane was used simultaneously for the application of NA to dermal skin vessels to test for local vasoconstriction and to collect interstitial fluid during the whole experiment. Vascular reactions to NA perfusion were tested indirectly by means of laser Doppler flowmetry and analysis of TPC in the dialysate, reflecting vascular permeability and capillary blood flow.

As shown before, ${ }^{12}$ TPC in the dialysate declined during the first 30 minutes of microdialysis after insertion of the membrane because of depletion of protein from the interstitial tissue surrounding the membrane. After approximately 30 minutes, a steady state with constant rates of plasma protein filtration and washout was reached. Any further increase or decrease in TPC in the dialysis fluid following NA perfusion reflected an increase or decrease in local blood flow, provided that vascular permeability remained unchanged. ${ }^{12}$

TPC did not differ between patients with FD and controls during steady state - that is, before NA application, as well as in response to NA application. The latter suggests that both groups were exposed to sufficiently high NA concentrations to obtain a maximum vasoconstriction at the site of NA infiltration.

In controls protein extravasation returned to baseline values within 30 minutes after NA application. In contrast, protein extravasation remained at low values in the patients. Perfusion of the microdialysis membrane with Ringer's solution washed out NA and gradually decreasing NA concentration led to redilatation of skin blood vessels. This vasodilatation allowed for an increase of protein extravasation to baseline concentrations. In contrast, plasma extravasation remained at a low concentration during washout of NA in the patient group. This time course suggests prolonged vasoconstriction following NA application in patients with FD and thus hypersensitivity to NA.

A dose response with application of several concentrations of NA would have been the most reliable way to test for local hypersensitivity, but because the duration of the experimental session of microdialysis with only one concentration was already about 150 minutes, we were not able to perform an extended test procedure in patients with FD. However, analysis of the blanching zone around the microdialysis membrane should provide appropriate information about the dose response to NA. In the patients with FD the area of skin blanching around the site of NA application-that is, the area of local vasoconstriction-was significantly larger than that in controls $(p<0.001)$. It can be assumed that NA diffuses from the microdialysis membrane to both sides of the membrane and that the concentration of the applied NA in the tissue is inversely correlated to the distance from the membrane. Therefore, a larger blanching zone in patients with FD indicates that skin vessels respond to lower tissue concentrations of NA than in controls. In controls, there was no response to consecutively lower NA tissue concentration at the same distance from the membrane. This provides further evidence that patients with FD are hypersensitive to NA.

During NA microdialysis, local SBF was assessed in both FD patients and controls by laser Doppler technique. In contrast to previous studies on SBF at the thorax in the midclavicular area, ${ }^{8}$ this study showed reduced SBF in FD patients at baseline. The discrepancy in baseline flow may be due to different density of sympathetic innervation, reflecting the more pronounced deficiency of sympathetic fibres in patients with FD at distal body regions. ${ }^{5}$ However, normalised SBF values did not differ between patients and controls. In both groups, SBF values decreased to $25 \%$ of baseline values.

This result may appear contradictory to the hypothesis of denervation hypersensitivity. However, SBF was reduced close 
to zero in patients with FD during NA application. Thus, the degree of vasoconstriction might have been underestimated. Moreover, the laser probe was attached above the microdialysis membrane at the skin area of maximum NA concentration. At this point, NA concentration might have been supramaximal and therefore identical vasoconstriction would have been produced in patients and controls. Thus, increased vasoconstriction can be judged more reliably based on time course and spatial extent rather than the local SBF value.

However, during the first five minutes of NA application ("wash-in phase"), SBF was already decreasing steeply at the application site in patients with FD; this was not found in controls. The differences between the groups were significant $(p=0.002)$. Since tissue concentrations of NA are considered to be low at that time, the difference in the time course of SBF decrease confirms the hypothesis of hypersensitivity, although the maximum SBF decrease after reaching higher tissue concentrations of NA was not different.

The more widespread and prolonged vasoconstriction in the patients with FD may be attributed to upregulation of adrenoceptor density or receptor sensitivity. Since the number of sympathetic nerve fibres containing these terminals is reduced in $\mathrm{FD}$, a reduction of NA reuptake into postganglionic presynaptic sympathetic vesicles may also have a major role. ${ }^{13}$ In FD reduced sympathetic innervation of the vasculature with a reduced number of NA containing terminals is observed. ${ }^{4}$ In patients with FD the rise in plasma catecholamines and orthostatic hypotension is inadequate during postural challenge, ${ }^{13}$ while supine catecholamine concentrations do not differ from those of healthy controls. ${ }^{14}{ }^{15}$ Orthostatic hypotension is ascribed to a reduction of neurones in sympathetic ganglia, ${ }^{2}$ as well as vasomotor nerve fibres.

Despite the sympathetic deficiency, patients with FD manifest episodic, excessive hypertension following stressful conditions. ${ }^{5}$ This response has been attributed to denervation hypersensitivity, since an exaggerated blood pressure increase has been observed with NA infusion, ${ }^{6}$ and excessive miosis occurred with conjunctival instillation of diluted methacholine?

Our findings support the assumption of catecholamine hypersensitivity in patients with FD. Denervation supersensitivity to catecholamines may compensate partially for the reduced number of endogenously available sympathetic nerve fibres and NA containing terminals. Decreased sympathetic supply is presumed to be the cause of diminished NA plasma concentrations $^{14}$ and for the failure of NA to increase appropriately in response to orthostatic challenge in patients with FD. ${ }^{16}$ During stressful situations, an increased release of catecholamines from sources other than peripheral sympathetic nerve fibres in the skin-that is, the adrenal glandmay be sufficient to induce an exaggerated response, with vasoconstriction and blood pressure increase, if the population of NA receptors at the peripheral vessels is upregulated or hypersensitive.

This hypersensitivity may account for the extreme hypertension associated with dysautonomic crises in patients with FD. Further studies of the NA dose relation should clarify whether an increased risk of hypertensive autonomic crises can be identified in an individual patient by means of analysing local vascular responses to NA applied by microdialysis. Furthermore, our results suggest that the method of microdialysis can be applied successfully as a research technique for the evaluation of peripheral denervation hypersensitivity and may thus also be useful in the assessment of patients with other types of autonomic failure.

\section{ACKNOWLEDGEMENTS}

The authors thank Dr Clive Brown PhD for his valuable help preparing the manuscript. The study was supported by unrestricted grants from the Dysautonomia Foundation, New York, and from SanofiSynthelabo, Germany.

.

Authors' affiliations

A Bickel, *M J Hilz, Neurologische Klinik, Universität

Erlangen-Nürnberg, Schwabachanlage 6, 91054 Erlangen, Germany

F B Axelrod, New York University Medical Center, 530 First Avenue,

Suite 9Q, New York, NY 10016, USA

M Schmelz, Institut für Physiologie I, Universität Erlangen-Nürnberg,

Universitätsstrasse 17, 91054 Erlangen, Germany

H Marthol, New York University Medical Center, 550 First Avenue,

Suite 7 W 11, New York, NY 10016, USA

*Also the Department of Pediatrics and Department of Neurology, New York University Medical Center

Competing interests: none declared

\section{REFERENCES}

1 Pearson J, Axelrod F, Dancis J. Trophic functions of the neuron. V. Familial dysautonomia. Current concepts of dysautonomia: neuropathological defects. Ann NY Acad Sci 1974;228:288-300.

2 Pearson J, Pytel BA. Quantitative studies of sympathetic ganglia and spinal cord intermedio-lateral gray columns in familial dysautonomia. $J$ Neurol Sci 1978;39:47-59

3 Pearson J, Pytel BA, Grover-Johnson N, et al. Quantitative studies of dorsal root ganglia and neuropathologic observations on spinal cords in familial dysautonomia. J Neurol Sci 1978;35:77-92.

4 Grover-Johnson N, Pearson J. Deficient vascular innervation in familia dysautonomia, an explanation for vasomotor instability. Neuropathol Appl Neurobiol 1976;2:217-24.

5 Axelrod FB, Nachtigal R, Dancis J. Familial dysautonomia: diagnosis, pathogenesis and management. Adv Pediatr 1974;21:75-96.

6 Smith A, Dancis J. Exaggerated response to infused norepinephrine in familial dysautonomia. N Engl J Med 1964;270:704-7.

7 Smith A, Hirsch J, Dancis J. Responses to infused metacholine in familial dysautonomia. Pediatrics 1965;36:225-30.

8 Weiser M, Hilz M, Bronfin L, et al. Assessing microcirculation in familial dysautonomia by laser Doppler flowmeter. Clin Auton Res 1998;8:13-23.

9 Slaugenhaupt SA, Blumenfeld A, Gill SP, et al. Tissue-specific expression of a splicing mutation in the IKBKAP gene causes familial dysautonomia. Am J Hum Genet 2001:68:598-605.

10 Schmelz M, Luz O, Averbeck B, et al. Plasma extravasation and neuropeptide release in human skin as measured by intradermal microdialysis. Neurosci Lett 1997;230:1 17-20.

11 Bradford MM. A rapid and sensitive method for the quantification of microgram quantities of protein utilizing the principle of protein-dye binding. Ann Biochem 1976;72:248-54.

12 Schmelz M, Zeck S, Raithel $M$, et al. Mast cell tryptase in dermal neurogenic inflammation. Clin Exp Allergy 1999;29:695-702.

13 Axelrod FB, Goldstein DS, Holmes C, et al. Genotype and phenotype in familial dysautonomia. Adv Pharmacol 1998:42:925-8.

14 Axelrod FB, Krey L, Glickstein JS, et al. Atrial natriuretic peptide response to postural change and medication in familial dysautonomia. Clin Auton Res 1994;4:311-8

15 Axelrod FB, Goldstein DS, Holmes C, et al. Pattern of plasma levels of catecholamines in familial dysautonomia. Clin Auton Res 1996:6:205-9.

16 Ziegler MG, Lake CR, Kopin IJ. Deficient sympathetic nervous response in familial dysautonomia. N Engl J Med 1976;294:630-3. 